\title{
State of the art vaccination strategies as primary prevention to reduce incidence of gastrointestinal cancers
}

\author{
Rhea Daniel ${ }^{1}$, Sarah Lowry ${ }^{1}$, Harpreet Pall $^{2}$ \\ ${ }^{1}$ Department of Pediatrics, St. Christopher's Hospital for Children, Philadelphia, PA, USA; ${ }^{2}$ Department of Pediatrics, K. Hovnanian Children's \\ Hospital/Hackensack Meridian Health School of Medicine at Seton Hall University, Nutley, NJ, USA \\ Contributions: (I) Conception and design: All authors; (II) Administrative support: None; (III) Provision of study materials or patients: None; (IV) \\ Collection and assembly of data: R Daniel, S Lowry; (V) Data analysis and interpretation: All authors; (VI) Manuscript writing: All authors; (VII) \\ Final approval of manuscript: All authors. \\ Correspondence to: Harpreet Pall. Chairman. Department of Pediatrics, K. Hovnanian Children's Hospital/Hackensack Meridian Health School of \\ Medicine at Seton Hall University, Nutley, NJ, USA. Email: Harpreet.Pall@hackensackmeridian.org.
}

\begin{abstract}
Immunizations have influenced the epidemiology of numerous gastrointestinal cancers. Human papillomavirus (HPV) is a common sexually transmitted infection (STI). Although most infections are transient and asymptomatic, persistent infections with oncogenic strains of HPV can progress to cervical, anal, penile, vaginal, vulvar, and oropharyngeal cancers. The introduction of HPV vaccinations has drastically reduced incidences of HPV-vaccine related infections and HPV related cervical cancers. The vaccine has proven to be safe and effective however, HPV vaccination rates have yet to reach target goals in the U.S. and many countries worldwide have not incorporated the vaccine into national immunization programs. The first successful nationwide vaccination program was employed against hepatitis B virus (HBV) in Taiwan in 1984 and demonstrated a statistically significant decrease in the incidence of hepatocellular carcinoma (HCC) in the 6 to 10 years after implementation of universal HBV vaccinations in infants. Twenty-year follow-up studies have continued to demonstrate statistically significant decreased rates of HBV related HCC among vaccinated populations. Despite the successful decrease in incidence of HBV-related HCC, efforts to create an effective prophylactic vaccination against hepatitis $\mathrm{C}$ virus (HCV) to prevent chronic $\mathrm{HCV}$ infection and its associated morbidity, including HCV-related HCC, have to date been unsuccessful.
\end{abstract}

Keywords: Vaccines; cervical cancer; hepatocellular carcinoma (HCC)

Submitted Dec 23, 2019. Accepted for publication Dec 31, 2019.

doi: 10.21037/jgo.2020.01.02

View this article at: http://dx.doi.org/10.21037/jgo.2020.01.02

\section{Introduction}

Primary prevention targets healthy individuals with a focus on disease prevention. Immunizations are a well-known form of primary prevention that has been used to prevent morbidity and mortality from numerous infectious agents. This review article focuses on immunizations that have influenced the epidemiology of cancer. Specifically, it will discuss vaccinations against human papillomavirus (HPV), hepatitis B virus (HBV), and hepatitis $\mathrm{C}$ virus (HCV) and their associated malignancies.

\section{HPV vaccination and gastrointestinal cancers}

\section{Overview}

HPV is a common sexually transmitted infection (STI) most frequently acquired after first sexual activity (1). HPV infects basal epithelial cells and most infections are transient and asymptomatic however, chronic infection with oncogenic strains of HPV can progress to cervical, anal, penile, vaginal, vulvar, and oropharyngeal cancers (2). The first Food and Drug Administration (FDA) approved HPV vaccine in the U.S. was a quadrivalent vaccine $(4 \mathrm{vHPV})$ 
targeting HPV strains 16, 18, 6, and 11 (3). It was first approved in 2006 with recommendations to vaccinate females aged 9 through 26 years of age (3). HPV 16 and 18 are responsible for causing $70 \%$ of cervical cancers in females and HPV 9 and 11 cause $90 \%$ of genital warts (3). HPV 16 and 18 are also responsible for $87 \%$ of anal cancers and $85 \%$ of cancers of the head and neck (4). In 2014, a 9-valent vaccine (9vHPV) was FDA approved (5). 9vHPV includes HPV strains covered by $4 \mathrm{vHPV}$ and has expanded coverage to include high-risk HPV types $31,33,45,52$, and 58 (5). This $9 \mathrm{vHPV}$ protects against an additional $14 \%$ of HPV-related cancers in the U.S. (5).

\section{History of HPV vaccination}

In the U.S. the $2 \mathrm{vHPV}$ and $4 \mathrm{vHPV}$ vaccines are no longer available due to low market demand and the 9vHPV vaccine is currently the standard of care. However, $2 \mathrm{vHPV}$ and $4 \mathrm{vHPV}$ are used in other countries around the world $(1,2)$. All 3 versions are intended to be administered prior to the onset of sexual activity when first exposure to HPV typically occurs (1). Studies investigating the immunogenicity of the $2 \mathrm{vHPV}$ and $4 \mathrm{vHPV}$ showed $2 \mathrm{vHPV}$, which uses a different adjuvant than $4 \mathrm{vHPV}$ and $9 \mathrm{vHPV}$, produced consistently higher levels of antibodies against HPV 16 and 18 after 60 months. However, the clinical relevance of this is unknown as no correlation with protection has been elucidated (1). Two randomized controlled trials have compared 3 doses of $4 \mathrm{vHPV}$ and $9 \mathrm{vHPV}$ with $4 \mathrm{vHPV}$ showing non-inferiority to $9 \mathrm{vHPV}$ for overlapping strains and 9vHPV showing high efficacy in preventing persistent infection against HPV types $31,33,45,52$, and 58 compared to $4 \mathrm{vHPV}$ (1). All 3 vaccines, $2 \mathrm{vHPV}, 4 \mathrm{vHPV}$, and $9 \mathrm{vHPV}$ have similar effectiveness in preventing cervical cancer (1).

The impact of HPV vaccination is readily evidenced by examining trends of HPV related infection in Australia. Australia implemented a national school-based, government funded HPV vaccination program targeting females aged 12 to 13 years old in 2007 (6). The program utilized $4 \mathrm{vHPV}$ and resulted in $73 \%$ of females aged $12-13$ years old receiving 3 doses of the vaccine (6). Studies have shown a $47.5 \%$ effectiveness of the $4 \mathrm{vHPV}$ against cervical intraepithelial neoplasia type 3 (CIN3) and adenocarcinoma in situ (AIS) in women who had received 3 doses of the vaccine and a $36.4 \%$ reduction in CIN3 and AIS for women who received any doses of the vaccine (6). A $77 \%$ reduction in vaccine-related HPV infections was also seen in women
18-24 years (6). A herd immunity effect was evidenced with reduced vaccine-related HPV prevalence in women in the same age cohort who were not vaccinated (6). Interestingly, there was also an increased prevalence of HPV types 31, 33, 35 , and 45 in unvaccinated females compared to vaccinated females which provides evidence that there is a crossprotective effect against HPV strains that are genetically similar to those included in the vaccine (6).

\section{HPV vaccination worldwide}

Despite the known efficacy of HPV vaccines in reducing the incidence of HPV-vaccine related infection and cancers and its demonstrated safety, as of March 2017 only 37\% of countries worldwide had incorporated HPV vaccines into their national immunization program for females and only $6 \%$ of countries incorporated HPV vaccination into their national immunization program for males (1). In the U.S., the Healthy People 2020 goal is to have $80 \%$ of adolescents aged 13-15 years of age fully vaccinated against HPV (7). As of 2016 vaccination rates in the U.S. for females and males between the ages of $13-17$ were $43 \%$ and $31.5 \%$ respectively (7).

\section{HPV vaccine besitancy}

Challenges regarding timely HPV vaccination include parental hesitancy. Studies have shown some parents believe HPV vaccinations will encourage earlier sexual activity or promiscuity (8). Recommendations for overcoming vaccine hesitancy include framing HPV vaccination discussions with families as an opportunity to prevent HPV related cancer deaths and starting the discussion regarding HPV vaccination at the same time as other mandated vaccines such as hepatitis B vaccine (8). Educating caregivers that the goal of vaccination is to achieve immunity prior to exposure can also help overcome this hesitancy. In the U.S. the Advisory Committee on Immunization Practices (ACIP) recommends HPV vaccination for children and adults 9-26 years of age with routine commencement at age 11 or 12 years old (2). For patients $27-45$ years old who are not adequately vaccinated and who have not been exposed to the virus before or who are at risk for infection or exposure to new HPV strains the vaccine may be given. However, it must be discussed that the vaccine will not eradicate, treat, decrease time to clearance, or prevent progression of HPV infections that have already been established (2). 


\section{HPV and oropharyngeal cancers}

Oropharyngeal cancer is located at the base of the tongue, the tonsils, soft palate and pharynx. In the past, oropharyngeal cancers were commonly located in the anterior oropharynx and associated with alcohol and tobacco use (9). Currently, posterior oropharyngeal cancers are more common with $30 \%$ of worldwide cases presumed to be HPV-related with higher prevalence in developing countries $(4,8,10)$. In the U.S., $70-90 \%$ of new cases of oropharyngeal cancer are confirmed as HPV-related invasive squamous cell carcinoma and are diagnosed in younger, healthier patients $(9,10)$. Compared with 30-50 years ago, sexual behaviors have changed considerably to younger ages of first sexual encounters, increasing number of partners, and more oral sexual acts $(9,11)$. With these changes, exposure to HPV is occurring at younger ages, leading to an increased risk of HPV oropharyngeal cancer.

In Australia, the incidence of HPV oropharyngeal cancers increased yearly from $18.6 \%$ in 1982 to $28.7 \%$ in 2005 with the majority of cancers located in tonsillar areas of the oropharynx (11). In the U.S. between 1988 and 2004 oropharyngeal cancers positive for HPV increased by $225 \%$ (12). HPV and oral cancer were first linked due to presence of juvenile respiratory papillomatosis in neonates exposed to maternal genital infections during birth (9). Approximately $7 \%$ of people in the U.S. have an oral HPV infection of any type at any time. Most of these infections clear within a year however, $20 \%$ may progress to cancer (9).

Although the HPV vaccine is not currently approved for prevention of oropharyngeal cancers, it is hypothesized that vaccination will cause a decline in new cases $(10,11)$. Little is known about the natural disease course of precancerous oral HPV infection progressing to oropharyngeal cancer (9). Early detection of oropharyngeal cancer is difficult because there are no identified precursor lesions (9). Due to a lack of routine surveillance methods for early detection, studies have not been designed to assess effectiveness of $\mathrm{HPV}$ vaccination for primary prevention of HPV-related oropharyngeal cancers. In a double-blind controlled trial in Costa Rica investigating HPV vaccination efficacy, oral secretions for HPV DNA by PCR were tested 4 years after vaccination. There was a $93 \%$ reduction in HPV oral infections in the vaccinated group compared to the control group indicative of possible protection with vaccination (13). However, oral HPV status was not assessed at the start of the study making it unclear if they were truly new cases acquired during the study period (13). Future studies for
HPV vaccination in prevention of oropharyngeal cancer needs to be done to investigate potential benefits of vaccination.

\section{HBV vaccination and hepatocellular carcinoma (HCC)}

\section{Overview}

HCC is the fifth most common cancer and the third leading cause of cancer mortality worldwide $(14,15)$. The incidence of HCC is unequally distributed among countries with the greatest burden of disease concentrated among developing regions. Mongolia has the highest incidence of HCC in the world and China accounts for $50 \%$ of all HCC cases (14). Globally, the leading cause of HCC is HBV with most HBV related HCC occurring in Asia and sub-Saharan Africa (14). Modes of transmission vary by region with vertical transmission commonly occurring in high endemic regions and horizontal transmission more common in low endemic regions (16). The risk of developing chronic HBV infection is inversely related to age with $80-90 \%$ of infants infected in the first year of life developing chronic HBV infection vs. $5 \%$ in healthy adult populations (16). Chronic carriers of HBV are 15 times more likely to develop HCC than noncarriers and it is estimated $60-80 \%$ of HCC worldwide is attributed to $\mathrm{HBV}(17,18)$. HBV is a vaccine-preventable disease and implementation of nationwide vaccination programs have decreased the incidence of chronic $\mathrm{HBV}$ and subsequent development of HCC, thus making HCC one of the first vaccine-preventable cancers.

\section{Nationwide vaccination programs \& impact on incidence of HCC}

Passive immunity via hepatitis B immune globulin (HBIG) was first proven effective in reducing perinatal transmission when given immediately following birth in 1983 in Taiwan (19). A randomized double-blind placebo-controlled trial showed three doses of HBIG had an efficacy between $71-87 \%$ in reducing development of persistent hepatitis B surface antigen (HBsAg) in infants (19). Later, studies conducted in Taipei showed the combined efficacy of HBV vaccination in conjunction with HBIG given one time immediately after birth had a combined efficacy of $94 \%$ in preventing persistent development of HBsAg in infants (20). The first nationwide HBV vaccination program was implemented in Taiwan in 1984 using combined passive and 
cell mediated immunity. Studies examining the incidence of HCC among children between 6-14 years old after the vaccination program was implemented showed a statistically significant decrease in the incidence of HCC from 0.52 per 100,000 prior to vaccination to 0.13 per 100,000 in the 6 to 10 years after implementing universal $\mathrm{HBV}$ vaccination (21). A 20-year follow-up study examined the incidence of HCC in patients aged 6-29 years old and found vaccinated cohorts still had a statistically significant reduced incidence of HCC compared to unvaccinated groups. This provided evidence that protective benefits of vaccination at birth extend into later adulthood (22). Currently the World Health Organization (WHO) recommends at least 3 doses of hepatitis B vaccine for all children worldwide (23). The first dose is considered timely if administered within 24 hours of birth (23). In 2016, 52\% of WHO represented countries had vaccine schedules that recommended universal HBV vaccination at birth (23). Only $19 \%$ of countries in the African region had universal $\mathrm{HBV}$ vaccination at birth compared to $93 \%$ of countries in the western Pacific region (23). Some of the barriers to achieving timely vaccination include the large percentage of home births in developing countries and misconceptions regarding vaccinating pre-term and low-birth weight infants. Certain HBV vaccines have been shown to be stable at temperatures up to 37 degrees Celsius for 1 month and at ambient temperatures up to 45 degrees Celsius for 1 week (23). The ability to store the vaccine at room temperature without the use of cold chain equipment would increase accessibility of the vaccine in many developing countries however, there has not yet been an $\mathrm{HBV}$ vaccine approved for ambient storage (23).

\section{Long-term immunity}

Research regarding how long after vaccination immunity to $\mathrm{HBV}$ persists is ongoing. The longest cohort study completed to date showed $94 \%$ of study participants vaccinated at 6 months of age or older exhibited evidence of protection out to 30 years after completing the primary vaccination series (24). No patients developed chronic $\mathrm{HBV}$ infection (24). A 35 -year study follow-up is planned. This study included children vaccinated at 6 months and older and did not include infants. However, a study conducted in Taiwan revealed $71 \%$ and $37.4 \%$ of patients vaccinated at birth had protective levels of anti-hepatitis B surface immune globulin (anti-HBs) present at 7 and 12 years of age respectively (25). This indicates that children vaccinated at birth have waning antibody levels compared to children vaccinated at an older age. The clinical significance of this is not clear as no study participants developed chronic HBV, which may be attributed to persistent immune memory that is able to provide protection against infection irrespective of serum anti-HBs levels (25). Current WHO guidelines do not recommend hepatitis $B$ booster vaccines as there is no evidence to support the efficacy of booster vaccines. Further studies are needed to evaluate life-long immunity and the potential need for booster vaccinations in certain subgroups (26).

\section{New advancements}

Research regarding the best vaccine and schedule for immunocompromised subgroups is ongoing. Immunocompromised adults over age 40 have shown hypo-responsiveness to aluminum adjuvants used in HBV vaccines (26). For these special populations $\mathrm{HBV}$ vaccine adjuvanted with aluminum phosphate and monophosphoryl lipid A is available to help stimulate a more robust immune response in immunocompromised patients (26). In 2017, the FDA approved a 2-dose vaccine, Heplisav-B, approved for use in patients 18 years and older which uses synthetic CpG motifs as an adjuvant (27). The use of CpG motifs stimulates the immune system via toll-like receptors and has been shown to result in substantially higher titer rates than the use of aluminum adjuvants in immunocompromised patients (27). The 2-dose vaccine can be completed in 4 weeks resulting in faster seroprotection than currently available vaccines (27).

\section{State of vaccination for hepatitis C}

\section{Overview}

Globally, HCV infection affects approximately 170 million people with an estimated global incidence of 3-4 million new cases every year (28). It is the leading cause of liver transplantation in the U.S., Australia, and most of Europe (29). Approximately $75-85 \%$ of patients will develop chronic $\mathrm{HCV}$ after a primary infection (29). Patients with $\mathrm{HCV}$ related liver cirrhosis have a $2-6 \%$ risk of developing HCC (28).

\section{Current treatment}

$\mathrm{HCV}$ is a curable disease. The standard of care for treatment historically involved the use of ribavirin and 
interferon alpha. Newer direct acting antivirals have also been developed with reduced length of antiviral treatment time and improved response rates. However, the costs of treatment regimens are extremely expensive and are accompanied by significant adverse effects (30). In addition, chronic HCV infections disproportionately affect patients living in developing countries who have limited access to medical care making the feasibility of lengthy treatment regimens requiring frequent follow-up difficult (28). After primary infection with $\mathrm{HCV}$, development of cirrhosis and HCC is an insidious process often occurring up to 20 years after initial infection (29). Many patients are unaware they are even infected with HCV until clinical symptoms or laboratory findings emerge. Primary prevention via vaccination against $\mathrm{HCV}$ is a highly researched area. Since not all patients who are infected with HCV develop chronic infection and a significant percentage of patients are able to naturally mount an immune response capable of eliminating $\mathrm{HCV}$, research efforts investigating mimicking this immune response is thought to be a realistic goal. However, despite extensive research efforts there is currently no effective prophylactic vaccine against $\mathrm{HCV}(31)$.

\section{Challenges of HCV vaccine development}

One of the challenges regarding development of an effective prophylactic vaccine against $\mathrm{HCV}$ involves the wide heterogeneity of the virus itself. HCV is a single stranded RNA virus of the Flaviviridae family with six distinct genotypes and over 100 subtypes (31). HCV undergoes a high rate of mutations during replication due to lack of a proof-reading mechanism of its viral polymerase and has a short half-life with a rapid turnover rate contributing to great genetic variability (31). This genetic inconsistency poses a challenge to finding a reliable target for vaccine development and aids the virus in evading hosts immune responses. Another challenge encountered in vaccine development has been the lack of in-vitro models to study the HCV life cycle and viral-host interactions. In 2005, the first tissue culture model based on recombinant cells allowed for reliable HCV replication in a human hepatoma cell line and provided a way to study these interactions (32). HCV has only been reliably reproduced in the hepatocytes of humans and chimpanzees making the chimpanzee the only immunocompetent in vivo animal model available for research (31). Clinical studies in humans are difficult due to the high incidence of $\mathrm{HCV}$ in vulnerable patient populations such as intravenous drug users in developed nations creating ethical dilemmas for testing (31).

Due to the virulence of $\mathrm{HCV}$ a live attenuated vaccination is not a current focus of vaccine development. Patients with chronic HCV infection display high titers of antibodies against $\mathrm{HCV}$ so it is unlikely that a vaccination inducing a humoral response alone will be protective against developing chronic HCV and subsequent HCC (31). Recombinant protein vaccines typically generate a humoral and cellular immune response against viral epitopes from isolated genes and many studies in animal models are currently underway using recombinant proteins $(31,33)$. The $\mathrm{HBV}$ vaccine was the first successful recombinant protein vaccine used in humans and is comprised of a hepatitis $B$ viral antigen that is conserved among the virus and induces a humoral response protective against infection (33). Since cellular immunity and the induction of cytotoxic $\mathrm{T}$ lymphocytes (CTLs) seems to be a critical component of achieving $\mathrm{HCV}$ control and HCV has more heterogeneity than HBV, this approach of inducing a humoral immune response using a conserved viral antigen has not been successful in HCV vaccine development. It is also part of the reason why an efficacious vaccination program against $\mathrm{HCV}$ similar to the one that exists for HBV has yet to be created. Vaccines targeted at the HCV core protein which is the most conserved region in HCV genotypes has been a goal in the past however, has been difficult due to lack of adequate T-cell responses to this protein (33).

DNA-based vaccines are another approach currently being researched. These vaccines tend to produce a weaker humoral immune response and stronger cellular immune response than recombinant protein vaccines (33). An advantage to DNA-based vaccines over recombinant protein vaccines include simplicity of producing polyvalent DNA vaccines compared to polyvalent vaccines comprised of multiple different purified proteins (33). Recombinant vaccines using defective viruses encoded with transgenic viral proteins have been shown to permit high levels of expression of antigenic proteins and induces a high frequency of CTL precursors (34).

\section{Previous HCV vaccine trial}

The first HCV prophylactic vaccine trial involving human participants was recently completed in 2019 and utilized defective simian adenovirus vectors and attenuated modified vaccinia Ankara vectors encoded with $\mathrm{HCV}$ genotype $1 \mathrm{~b}$ non-structural polyproteins $(35,36)$. The study conducted 
by the National Institute of Allergy and Infectious Diseases was a double-blind randomized, placebo-controlled trial investigating the efficacy of a vaccine in preventing chronic $\mathrm{HCV}$ infection in high-risk patients between the ages of 18 and 45 years (36). Patients did not have evidence of $\mathrm{HCV}$ infection at time of enrollment in the study and were deemed high-risk for HCV infection due to recent drug use within the past 90 days (36). The vaccine developed for the study was composed of a two-dose vaccine regimen given 56 days apart (36). The first vaccine was created from chimpanzee adenovirus with non-structural proteins of HCV genotype $1 \mathrm{~b}$ (36). The second vaccine was composed of an attenuated Modified Vaccinia Ankara vector given as a booster (36). A control group received two placebo vaccinations also 56 days apart. The participants were monitored 18 months after completion of the vaccination series. Participants who developed HCV infection were then followed for an additional 9 months to determine chronic $\mathrm{HCV}$ infection which was defined as persistent viremia for at least 6 months following initial detection of the virus (36). Preliminary results of the study showed there was no difference in rates of chronic HCV infection between intervention and placebo groups and the vaccine was thus ineffective (see ClinicalTrials.gov NCT01436357).

\section{Future directions}

Although an effective prophylactic $\mathrm{HCV}$ vaccine has yet to be developed there are multiple areas of ongoing research into the development of a successful vaccine. Primary prevention against infectious oncogenic virus such as $\mathrm{HPV}$ and HBV have proven the feasibility of reducing the incidence of cancer through prophylactic vaccination. $\mathrm{HCV}$ poses unique challenges in vaccine development with much information regarding the development of chronic $\mathrm{HCV}$ infection and immune evasion remaining unknown.

\section{Conclusions}

Over the past 40 years, introduction of vaccinations against $\mathrm{HBV}$ and more recently HPV, have drastically decreased rates of vaccine-related infections and associated complications including gastrointestinal cancers. Despite the safety and effectiveness of HPV vaccines, vaccination rates are still not at target goals for the U.S. and many countries have not incorporated the vaccination into recommended vaccine schedules. Numerous studies have demonstrated the protective effect of early HPV vaccination against the development of CIN and AIS leading to subsequent decreases in HPV related cervical cancers. Now, with increasing trends of HPV-related gastrointestinal cancers such as oropharyngeal cancers, fully understanding the protective effects of early vaccination will be beneficial for expanding vaccine indications.

HCC is the fifth most common cancer and the third leading cause of cancer mortality globally $(14,15)$. HBV and HCV infections are known risk factors for the development of HCC. National HBV vaccine programs have proven cost-effective at preventing infection and drastically decreased rates of HBV associated HCC. With the success of $\mathrm{HBV}$ vaccination, several attempts have been made to develop an effective prophylactic $\mathrm{HCV}$ vaccine with hopes of reducing chronic HCV infections and preventing development of HCV related HCC. However, due to the unique characteristics of the virus itself no successful prophylactic vaccine has been created to date. Future directions continue to focus on vaccine development to extend primary protection against chronic $\mathrm{HCV}$.

\section{Acknowledgements}

Funding: None.

\section{Footnote}

Provenance and Peer Review: This article was commissioned by the Guest Editors (John F. Gibbs and Quyen D. Chu) for the series "Global GI Malignancies" published in fournal of Gastrointestinal Oncology The article has undergone external peer review.

Conflicts of Interest: All authors have completed the ICMJE uniform disclosure form (available at http://dx.doi. org/10.21037/jgo.2020.01.02). The series "Global GI Malignancies" was commissioned by the editorial office without any funding or sponsorship. The authors have no other conflicts of interest to declare.

Ethical Statement: The authors are accountable for all aspects of the work in ensuring that questions related to the accuracy or integrity of any part of the work are appropriately investigated and resolved.

Open Access Statement: This is an Open Access article distributed in accordance with the Creative Commons 
Attribution-NonCommercial-NoDerivs 4.0 International License (CC BY-NC-ND 4.0), which permits the noncommercial replication and distribution of the article with the strict proviso that no changes or edits are made and the original work is properly cited (including links to both the formal publication through the relevant DOI and the license). See: https://creativecommons.org/licenses/by-ncnd/4.0/.

\section{References}

1. World Health Organization. Human papillomavirus vaccines: WHO position paper, May 2017-Recommendations. Vaccine 2017;35:5753-5.

2. Meites E, Szilagyi PG, Chesson HW, et al. Human papillomavirus vaccination for adults: updated recommendations of the Advisory Committee on Immunization Practices. MMWR Morb Mortal Wkly Rep 2019;68:698-702.

3. Braaten KP, Laufer MR. Human papillomavirus (HPV), HPV-related disease, and the HPV vaccine. Rev Obstet Gynecol 2008;1:2-10.

4. de Martel C, Plummer M, Vignat J, et al. Worldwide burden of cancer attributable to HPV by site, country and HPV type. Int J Cancer 2017;141:664-70.

5. Bernstein HH, Bocchini JA Jr; COMMITTEE ON INFECTIOUS DISEASES. The Need to Optimize Adolescent Immunization. Pediatrics 2017;139:e20164186.

6. Garland SM. The Australian experience with the human papillomavirus vaccine. Clin Ther 2014;36:17-23.

7. Rodriguez AM, Do TQN, Goodman M, et al. Human papillomavirus vaccine interventions in the U.S.: a systematic review and meta-analysis. Am J Prev Med 2019;56:591-602.

8. Thomas TL, Strickland O, Diclemente R, et al. An opportunity for cancer prevention during preadolescence and adolescence: stopping human papillomavirus (HPV)related cancer through HPV vaccination. J Adolesc Health 2013;52:S60-8.

9. Guo T, Eisele DW, Fakhry C. The potential impact of prophylactic human papillomavirus vaccination on oropharyngeal cancer. Cancer 2016;122:2313-23.

10. Auluck A, Hislop G, Bajdik C, et al. Trends in oropharyngeal and oral cavity cancer incidence of human papillomavirus (HPV)-related and HPV-unrelated sites in a multicultural population: the British Columbia experience. Cancer 2010;116:2635-44.

11. Hocking JS, Stein A, Conway EL, et al. Head and neck cancer in Australia between 1982 and 2005 show increasing incidence of potentially HPV-associated oropharyngeal cancers. Br J Cancer 2011;104:886-91.

12. Gillison ML, Broutian T, Pickard RK, et al. Prevalence of oral HPV infection in the United States, 2009-2010. JAMA 2012;307:693-703.

13. Herrero R, Quint W, Hildesheim A, et al. Reduced prevalence of oral human papillomavirus (HPV) 4 years after bivalent HPV vaccination in a randomized clinical trial in Costa Rica. PLoS One 2013;8:e68329.

14. White DL, Kanwal F, Jiao L, et al. Epidemiology of hepatocellular carcinoma. In: Carr BI. Hepatocellular Carcinoma. Cham: Springer, 2016:3-24.

15. Schütte K, Bornschein J, Malfertheiner P. Hepatocellular carcinoma--epidemiological trends and risk factors. Dig Dis 2009;27:80-92.

16. Gomes C, Wong RJ, Gish RG. Global perspective on hepatitis B virus infections in the era of effective vaccines. Clin Liver Dis 2019;23:383-99.

17. Song C, Lv J, Liu Y, et al. Associations between hepatitis $B$ virus infection and risk of all cancer types. JAMA Netw Open 2019;2:e195718.

18. Chang MH. Hepatitis B virus and cancer prevention. Recent Results Cancer Res 2011;188:75-84.

19. Beasley RP, Hwang LY, Stevens CE, et al. Efficacy of hepatitis B immune globulin for prevention of perinatal transmission of the hepatitis B virus carrier state: final report of a randomized double-blind, placebo-controlled trial. Hepatology 1983;3:135-41.

20. Beasley RP, Hwang LY, Lee GC, et al. Prevention of perinatally transmitted hepatitis $B$ virus infections with hepatitis B immune globulin and hepatitis B vaccine. Lancet 1983;2:1099-102.

21. Chang MH, Chen CJ, Lai MS, et al. Universal hepatitis B vaccination in Taiwan and the incidence of hepatocellular carcinoma in children. Taiwan Childhood Hepatoma Study Group. N Engl J Med 1997;336:1855-9.

22. Chang MH, You SL, Chen CJ, et al. Decreased incidence of hepatocellular carcinoma in hepatitis B vaccinees: a 20-year follow-up study. J Natl Cancer Inst 2009;101:1348-55.

23. Implementation of hepatitis B birth dose vaccination worldwide, 2016. Wkly Epidemiol Rec 2018;93:61-72.

24. Bruce MG, Bruden D, Hurlburt D, et al. Antibody levels and protection after hepatitis $\mathrm{B}$ vaccine: results of a 30year follow-up study and response to a booster dose. J Infect Dis 2016;214:16-22.

25. Lin YC, Chang MH, Ni YH, et al. Long-term immunogenicity and efficacy of universal hepatitis B virus 
vaccination in Taiwan. J Infect Dis 2003;187:134-8.

26. World Health Organization. Hepatitis B vaccines: WHO position paper, July 2017 - Recommendations. Vaccine 2019;37:223-5.

27. Splawn LM, Bailey CA, Medina JP, et al. Heplisav-B vaccination for the prevention of hepatitis $B$ virus infection in adults in the United States. Drugs Today (Barc) 2018;54:399-405.

28. de Oliveria Andrade LJ, D'Oliveira A, Melo RC, et al. Association between hepatitis $\mathrm{C}$ and hepatocellular carcinoma. J Glob Infect Dis 2009;1:33-7.

29. Chen SL, Morgan TR. The natural history of hepatitis $C$ virus (HCV) infection. Int J Med Sci 2006;3:47-52.

30. Feeney ER, Chung RT. Antiviral treatment of hepatitis C. BMJ 2014;348:g3308.

31. Halliday J, Klenerman P, Barnes E. Vaccination for hepatitis $\mathrm{C}$ virus: closing in on an evasive target. Expert

Cite this article as: Daniel R, Lowry S, Pall H. State of the art vaccination strategies as primary prevention to reduce incidence of gastrointestinal cancers. J Gastrointest Oncol 2021;12(Suppl 2):S316-S323. doi: 10.21037/jgo.2020.01.02
Rev Vaccines 2011;10:659-72.

32. Stoll-Keller F, Barth H, Fafi-Kremer S, et al. Development of hepatitis $\mathrm{C}$ virus vaccines: challenges and progress. Expert Rev Vaccines 2009;8:333-45.

33. Forns X, Bukh J, Purcell RH. The challenge of developing a vaccine against hepatitis C virus. J Hepatol 2002;37:684-95.

34. Arribillaga L, de Cerio AL, Sarobe P, et al. Vaccination with an adenoviral vector encoding hepatitis $C$ virus (HCV) NS3 protein protects against infection with $\mathrm{HCV}$ recombinant vaccinia virus. Vaccine 2002;21:202-10.

35. Swadling L, Capone S, Antrobus RD, et al. A human vaccine strategy based on chimpanzee adenoviral and MVA vectors that primes, boosts, and sustains functional HCVspecific T cell memory. Sci Transl Med 2014;6:261ra153.

36. Bailey JR, Barnes E, Cox AL. Approaches, progress, and challenges to hepatitis $\mathrm{C}$ vaccine development. Gastroenterology 2019;156:418-30. 